\title{
Rheumatic Diseases - What Influence do they Really Have on the Incidence of Periprothethic Joint Infections after Total Knee Arthroplasty?
}

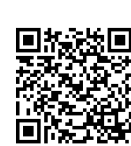

\author{
Stella Oberberg*, Leonard Ulbrich, Jan Nottenkamper, Jan Krapp and Roland E Willburger \\ Department of Orthopaedic Surgery, Katholisches Klinikum Bochum, Martin-Luther-Hospital, Germany
}

Submission: April 14, 2021; Published: April 23, 2021

*Corresponding author: Stella Oberberg, Department of Orthopaedic Surgery, Katholisches Klinikum Bochum, Martin-Luther-Hospital, Voedestrasse 79, 44866 Bochum, Germany

\section{Abstract}

Introduction: Periprosthetic joint infections (PJI) are serious complications after total knee arthroplasty (TKA). According to literature predisposing diseases, operation-specific and postoperative factors are important in development of PJIs. There are still divergent positions in the literature regarding the significance of individual risk factors. The aim of this study was to determine the incidence of PJI in our institution from 2008 to 2018, to identify risk factors by comparing affected patients with a normal population.

Method: Fifteen PJIs were detected during research period. Data of 501 consecutive patients were collected retrospectively for comparison A matched-pair analysis was performed in addition to an analysis of categorical and metric characteristics of the total population ( $\mathrm{n}=516)$ to adjust the unequal strengths of both groups.

Results: The incidence of PJI was $0.8 \%$ in our institution. Analysis of all patients showed significant correlations of PJI regarding blood transfusion, hematoma formation and postoperative urinary tract infection. The presence of renal insufficiency, nicotine consumption, and prolonged duration of surgery were identified as risk factors. After matched-pairs analysis (MPA), prolonged surgical duration, blood transfusion and preoperatively decreased haemoglobin (HB) were confirmed as independent risk factors. There was no significant difference regarding the presence of rheumatic diseases.

Discussion: Determining risk factors were preoperative anaemia, blood transfusion and prolonged operation times. The presence of rheumatic disease did not appear to be a risk factor. These findings should be incorporated into surgical preparation. For infection prophylaxis, preoperative HB elevation and rapid surgical procedures with low intraoperative blood loss should be discussed.

Keywords: Periprosthetic infections; Total knee arthroplasty; Prophylaxis; Risk factors; Anaemia, Duration of surgery; Blood transfusion; Rheumatoid arthritis

Abbreviations: ASA: American Society of Anaesthesiologists; HB: Haemoglobin; BMI: Body-Mass-Index; DMARD: Disease-modifying AntiRheumatic Drug; IP: Infection-Population; MPA: Matched-Pairs Analysis; NP: Normal-Population; PJI: Periprosthetic Joint Infections; TKA: Total knee Arthroplasty

\section{Introduction}

Implantation of a total knee arthroplasty (TKA) is one of the most common operations in orthopaedics. The number of TKA is steadily increasing due to demographic changes in society [1]. The majority of patients (over 85 percent) present good to very good results after TKA [2]. However, a significant decrease in revision surgeries has not yet been observed despite improved surgical techniques and innovative implants with long prosthesis service lives. Schwartz et al. [3] even predict an increasing number of complications with an increased need for second operations of 170 percent by the year 2030. Early complications include periprosthetic infections in addition to aseptic loosening and dislocation and periprosthetic fractures [4]. The rates of infection after primary TKA are inconsistent in the literature. Despite increasing prevention strategies, the incidence of infection is reported to be 1-2 percent in recent publications [5,6].

Various patient-dependent and independent risk factors are discussed in the literature. Patient-dependent factors include preexisting conditions such as diabetes mellitus, obesity, rheumatoid arthritis, and nicotine and alcohol abuse. Patients with diabetes mellitus show worse outcomes after surgical intervention 
with prolonged inpatient stays and increased mortality [7]. Existing obesity is not only a risk factor for the development of osteoarthritis, but also a risk factor for postoperative complications. Thus, obese patients tend to have poorer wound healing, wound dehiscence, prolonged secretion, and increased hematoma formation [8]. According to literature, regular nicotine use causes peripheral vasoconstriction and associated tissue hypoxia due to activation of the sympathetic nervous system [9]. This results, among other things, in slower wound healing with an increased incidence of periprosthetic complications [10]. In their retrospective work, Crowe et al. identified nicotine abuse within one month before surgery as an independent risk factor for periprosthetic infections after primary knee arthroplasty [11]. Some authors also describe an association between the presence of renal insufficiency and an increased risk of PJI $[12,13]$. Regular consumption of alcohol is also associated with an increase in complications after prosthetic implantation [14,15]. Increased complication rates and infection rates have also been repeatedly described in patients with rheumatoid arthritis [16]. It is assumed that, in addition to the influence of immunomodulatory therapy, T-cell dysfunction is partly responsible [17].

The aim of the present study was to determine the frequency of periprosthetic infections in our institution over a period of 10 years. The focus was on detecting risk factors for periprosthetic infection after TKA. The supposed risk factors were compared with the results of a control group without periprosthetic infection. Supposed risk factors were: Elevated Score of the Physical Status System "American Society of Anaesthesiologists" (ASA), diabetes mellitus, obesity, history of malignancy, preoperative anaemia, and comorbidities such as rheumatoid arthritis or renal insufficiency. In addition, the influence of surgical time, surgeon experience, blood loss during surgery, anaesthetic procedure, and administration of foreign blood were observed. Other abnormalities such as prolonged wound secretion, electrolyte imbalances, and postoperative urinary tract infections were also considered in the study.

\section{Materials and Methods}

\section{Patients}

The data collection for this study was performed retrospectively by evaluating patient data from our department. For the research, the respective patient files as well as digital documents in the information management system "Orbis“ were accessed. All existing documents such as physician's reports, admission notes, operation reports, anaesthesia protocols, laboratory findings, microbiological findings, and medical course documentation were considered. The period under investigation was 2008 to 2018. In the years mentioned, a total of 15 patients with periprosthetic infection after implantation of primary knee arthroplasty were registered in our department. These patients constitute the „infection-population“ (IP) in the present study. The date of the primary implantation of the first included patient of this group was in May 2008 and that of the last in April 2018. The control-group consists of 501 patients who also received a primary TKA in the same department and did not develop a periprosthetic infection ("normal-population"; NP). The surgeries of this population were performed between January 2016 and December 2018, with a consecutive data collection backwards from 2018.

\section{Statistics}

The data collection was performed with "Microsoft Excel", "Stata/IC 16.1 for Unix" was used for statistical analysis. For descriptive analysis, patient data were categorized into categorical (such as prior surgery) and metric characteristics (such as patient age). Fisher's exact test was used to test whether group membership and the corresponding characteristic were independent. Whether the groups differed with respect to the distribution of a characteristic was tested with the Mann Whitney $\mathrm{U}$ test. To eliminate the influence of the large difference in the number of cases in the two groups (infection group and normal group) and to detect the significance of the results, an additional matched-pairs analysis (MPA) was performed. Matching criteria were gender, presence of diabetes, body-mass-index (BMI) $(<40 / \geq 40)$, ASA $(\leq 2 />2)$, and age $( \pm 2$ years). The comparison of the groups was performed by McNemar's exact test for the categorical characteristics and the Wilcoxon signed-rank test for connected samples. All statistical tests were performed at a significance level of 0.05 .

\section{Clinical examination and questioning}

For the evaluation, general data such as patient name, age, and gender as well as previous operations on the affected knee joint were recorded. Furthermore, patient-related data were collected. This included height, weight, body mass index, and alcohol and nicotine abuse. Comorbidities such as malignant diseases, relevant cardiovascular diseases, diabetes mellitus, diseases of the respiratory tract, liver and kidney were recorded. Special attention was paid to rheumatic diseases or other inflammatory systemic diseases and associated medication with immunomodulatory drugs or anticoagulants. The surgery-dependent-factors included the indication-related diagnosis, whereby a differentiation was made between primary and secondary arthrosis and inflammatory diseases. In addition, the duration of the operation, the side location, and whether the operation was performed by an experienced surgeon or a surgeon in training were recorded. In addition, the type of anaesthesia and antibiotic prophylaxis as well as the ASA classification were documented.

\section{Results}

The NP included more female than male patients $(69.7 \%$ versus $30.3 \%$ ). In contrast, an almost equal gender distribution could be observed in the IP. This difference between the groups turned out to be statistically insignificant. 


\section{Pre-existing Conditions}

Regarding to pre-existing diseases or conditions, only the presence of renal insufficiency $(p=0.021)$ and nicotine abuse $(p=0.047)$ showed significant differences in the group comparison. Renal insufficiency showed the largest percentage difference of all surveyed pre-existing conditions. In the infection group, 27 percent ( 4 of 15) reported having renal insufficiency. In comparison, only 7 percent (35 of 501) of patients in the normal group reported renal insufficiency. Patients were considered smokers if they were current smokers or reported having smoked regularly for at least one year in the past. The type of rheumatic disease included in the study was rheumatoid arthritis, spondylarthritis, and polymyalgia rheumatica. In the infectious group, one of five showed a rheumatoid disease, in the comparison group only one in ten. However, the difference between the two groups regarding the presence of a rheumatic disease was not found to be significant $(\mathrm{p}=0.170)$. There were also no significant differences between the two groups regarding previous operations on the affected knee joint $(\mathrm{p}=1.000)$ and the presence of diabetes mellitus $(\mathrm{p}=0.158)$ as well as other previous diseases (cardiovascular, respiratory diseases, etc.) (Table 1).

Table 1: Comparison of pre-existing conditions of the populations.

\begin{tabular}{|c|c|c|c|c|}
\hline Condition & choice & NP & IP & p-value \\
\hline \multirow{2}{*}{ Diabetes } & no & $10(66,7 \%)$ & $415(82,8 \%)$ & \multirow{2}{*}{0,158} \\
\hline & yes & $5(33,3 \%)$ & $86(17,2 \%)$ & \\
\hline \multirow{2}{*}{ Renal disease } & no & $11(73,3 \%)$ & $466(93,0 \%)$ & \multirow{2}{*}{$0,021 *$} \\
\hline & yes & $4(26,7 \%)$ & $35(7,0 \%)$ & \\
\hline \multirow{2}{*}{ Rheumatic disease } & no & $454(90,6 \%)$ & $12(80 \%)$ & \multirow{2}{*}{0,17} \\
\hline & yes & $47(9,4 \%)$ & $3(20,0 \%)$ & \\
\hline \multirow{2}{*}{ Previous operation } & no & $310(61,9 \%)$ & $9(60 \%)$ & \multirow{2}{*}{1} \\
\hline & yes & $191(38,1 \%)$ & $6(40 \%)$ & \\
\hline \multirow{2}{*}{ Nicotin-consumption } & no & $433(86,4 \%)$ & $10(66,7 \%)$ & \multirow{2}{*}{$0,047^{*}$} \\
\hline & yes & $68(13,6 \%)$ & $5(33,3 \%)$ & \\
\hline \multirow{3}{*}{ Alcohol-consumption } & no & $365(72,9 \%)$ & $8(53,3 \%)$ & \multirow{3}{*}{0,187} \\
\hline & regular & $54(10,8 \%)$ & $3(20 \%)$ & \\
\hline & irregular & $82(16,4 \%)$ & $4(26,7 \%)$ & \\
\hline \multicolumn{5}{|c|}{${ }^{*}$ statistically significant } \\
\hline
\end{tabular}

In the MPA, no significant difference could be found for any of the above-mentioned conditions (renal disease $p=0.083$; rheumatic disease $\mathrm{p}=0.655$; nicotine consumption $\mathrm{p}=0.083$ ), so that the preoperative patient findings in our collective are considered negligible regarding the incidence of periprosthetic infection (Figure 1).

\section{Duration of surgery}

A statistically highly significant difference $(\mathrm{p}<0.001)$ was provided by the duration of surgery, which was significantly longer in the infection-population (mean 91.9 minutes) than in the normal-population (mean 70.3 minutes). The IP showed a minimum of 68 and a maximum of 114 minutes. In the NP, the minimum surgery duration was 43 minutes, while the maximum surgery duration was 115 minutes. The examination of the surgery duration also showed the highest significance of all factors examined in the MPA ( $p=0.037)$ (Figure 2).

\section{Postoperative conditions}

The groups differed most regarding the postoperative course. There was a highly significant correlation of periprosthetic infections with receipt of blood transfusions $(p<0.001)$, hematoma formation $(\mathrm{p}=0.001)$, and proven urinary tract infection ( $\mathrm{p}=0.008)$. In the IP, 40 percent of patients received a blood transfusion. Comparatively, in the NP, transfusions occurred at a strikingly low rate of 3.6 percent. Thus, in the infectionpopulation, the rate of transfusions was three times higher than in the normal-population ( $40 \%$ versus $3.6 \%$ ). In the MPA, the transfusion of blood also showed a significant difference between the groups $(p=0.046)$. Regarding to this, the preoperative HB value also showed a significant difference between the two groups in the MPA ( $p=0.049)$. The formation of hematomas of significant size and other wound healing disorders was documented in seven cases. Three of these cases were in the IP and four cases in the 


\section{Orthopedics and Rheumatology Open Access Journal (OROAJ)}

NP (20\% of infections versus $0.8 \%)$. However, MPA revealed no significant difference between the collectives in this regard $(\mathrm{p}=0.83)$. Approximately one-quarter $(26.7 \%)$ of patients in the infection-group developed a urinary tract infection in the postoperative setting. In the normal-collective, it affected only about one in twenty (5.2\%). However, the defined statistical significance level could not be reached in the MPA in this case either (Figure 3).
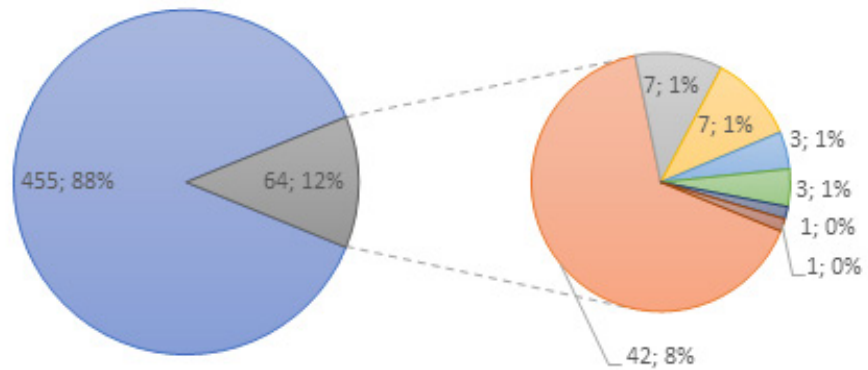

ano

口rheumatoid arthr itis

upsor iatic arthritis

afbromyalgia

ankylosing spondy litis

口 lupus erythematosus

口polymyagia arteriitica

apolymyagia rheumatica

Figure 1: Distribution of rheumatic diseases in the entire population.

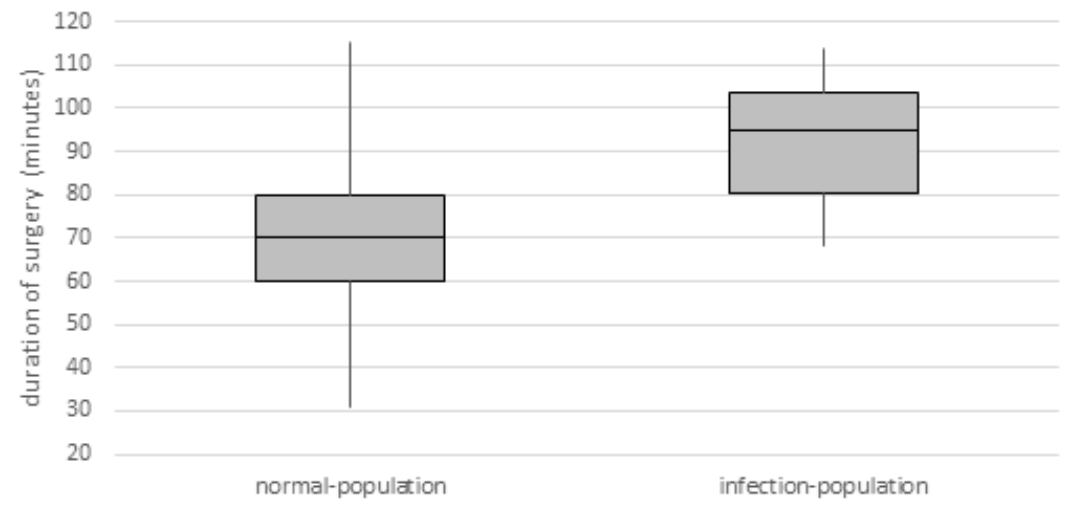

Figure 2: Comparison of the collectives related to the duration of surgery.

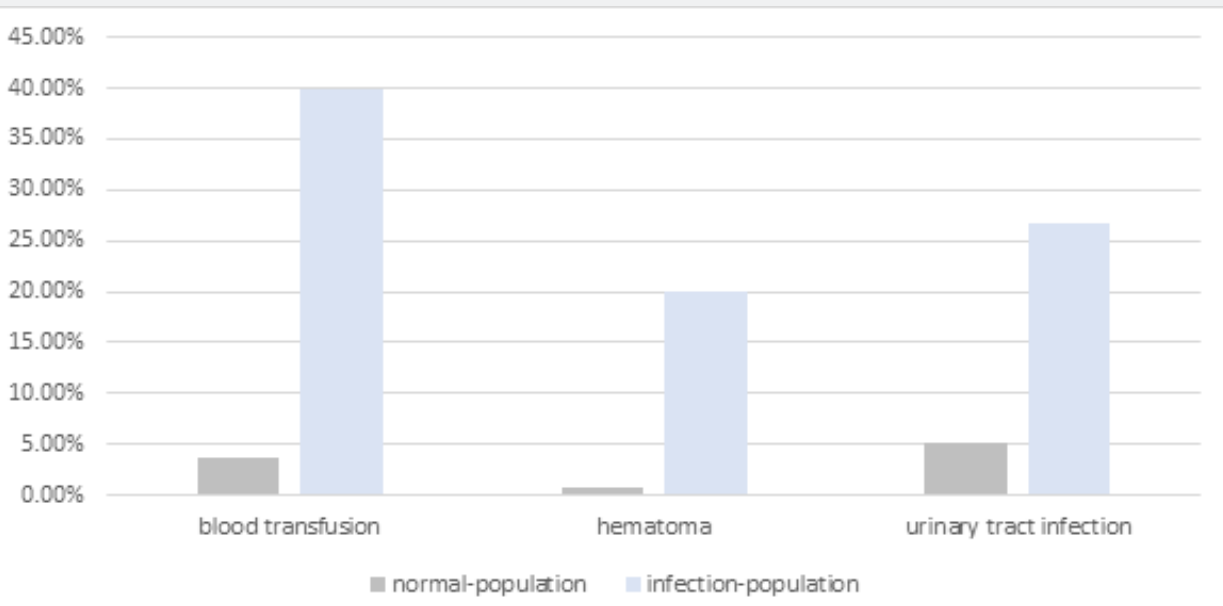

Figure 3: Comparison of postoperative conditions. 


\section{Discussion}

The presence of rheumatologic disease has been described by some authors as a possible risk factor for periprosthetic infection $[12,16,18]$, with continued "disease-modifying anti-rheumatic drug" (DMARD) therapy in particular being discussed as a crucial risk factor [18]. In addition, there is competing literature that does not assign an increased risk for PJI to rheumatoid arthritis patients without immunosuppressive therapy [5,19]. In our population, we did not find a statistically significant association between the presence of rheumatic disease and an accumulation of PJI. In relative terms, the infection-population included two times more patients with rheumatic disease compared with the normal-population, but without reaching statistical significance. Regarding therapy with DMARD and biologics, the recommendations for perioperative procedures of the „German Society of Rheumatology" were strictly applied in our population [20]. This might have been one reason why no significant association could be found. All other preoperative conditions such as nicotine abuse or the presence of renal insufficiency also failed statistical significance in the MPA, so that we could not confirm these conditions as risk factors in our population. On the other hand, a prolonged operation time turned out to be an independent risk factor with sufficient significance in our collective in the MPA. Wang et al. [21] were also able to identify prolonged surgery time as a risk factor in their study, showing that a 20 -minute increase in surgery time resulted in a $25 \%$ increase in the probability of infection.

In our study, the administration of foreign blood also resulted in significant differences between the two groups in both the overall analysis and after pairing. In the literature, studies have also demonstrated an increased incidence of infections after blood transfusion [22,23]. Pulido et al. [6] calculated a 2.1fold increased risk for PJI following the transfusion of blood. In summary, it can be stated for our collective that both a low preoperative HB value as described by Müller et al. [24] and the necessity of blood transfusion represent an independent risk factor for a PJI, so that the securing of a sufficiently high preoperative $\mathrm{HB}$ value should be given special importance in the surgical preparations. Furthermore, a high intraoperative blood loss can be avoided by a rapid and blood-saving surgical procedure, if necessary, with the use of antifibrinolytic drugs. While Pulido et al. [6] identified urinary tract infection as an independent risk factor for PJIs, Koulouvaris et al. did not show an increased rate of deep wound infections associated with urinary tract infections [25]. In our collective, postoperative urinary tract infection increased the risk of PJI significantly. However, after matching, statistical significance could not be confirmed, so we cannot consider the presence of a urinary tract infection as an independent risk factor in our study. According to the literature, the formation of a hematoma is also associated with an increased rate of wound infections [26], although sufficient significance was not achieved here in some studies [6,27]. In our population, this observation can be confirmed, as also here, after attenuating the confounding effects, the significance value decreased below the defined significance level. The main limiting factor of our study might be the retrospective study design, so that a causal association between the risk factors and the infection rate cannot be proven. Some confounders could be attenuated in the study using a matched-pair analysis; nevertheless, other confounders may have influenced the results.

\section{Conclusion}

In our sample, the most important risk factors for the incidence of periprosthetic infection were preoperative anaemia with HB values $<11.5 \mathrm{mg} / \mathrm{dl}$, the application of blood transfusions, and prolonged surgery times. These are all factors that can be considered during surgical preparation and argue for a possible need to raise the preoperative HB level. Furthermore, surgery should be as short as possible and thus probably less bloody. In our collective, there was no evidence for an increased risk in the presence of rheumatic disease under strict adherence to the recommendations of the German Society of Rheumatology on the perioperative management of DMARD and biologicals therapy in inflammatory rheumatic diseases.

\section{References}

1. Kurtz S, Mowat F, Ong K, Chan N, Lau E, et al. (2005) Prevalence of primary and revision total hip and knee arthroplasty in the United States from 1990 through 2002. J Bone Joint Surg Am 87(7): 14871497.

2. Lüring H, Bäthis H, Grifka J (2006) Bicondylar knee arthroplasty indication, technique, results and new developments. OP-Journal 22: 184-189.

3. Schwartz AM, Farley KX, Guild GN, Bradbury TL (2020) Projections and Epidemiology of Revision Hip and Knee Arthroplasty in the United States to 2030. J Arthroplasty 35(6): S79-S85.

4. Del Pozo JL, Patel R (2009) Clinical practice. Infection associated with prosthetic joints. N Engl J Med 361: 787-794.

5. Jämsen E, Huhtala H, Puolakka T, Moilanen T (2009) Risk factors for infection after knee arthroplasty. A register-based analysis of 43,149 cases. J Bone Joint Surg Am 91(1): 38-47.

6. Pulido L, Ghanem E, Joshi A, Purtill JJ, Parvizi J (2008) Periprosthetic joint infection: the incidence, timing, and predisposing factors. Clin Orthop Relat Res 466: 1710-1715.

7. Mraovic B, Suh D, Jacovides C, Parvizi J (2011) Perioperative hyperglycemia and postoperative infection after lower limb arthroplasty. J Diabetes Sci Technol 5(2): 412-418.

8. Patel VP, Walsh M, Sehgal B, Preston C, De Wal H, et al. (2007) Factors associated with prolonged wound drainage after primary total hip and knee arthroplasty. J Bone Joint Surg Am 89(1): 33-38. 
9. Jensen JA, Goodson WH, Hopf HW, Hunt TK (1991) Cigarette smoking decreases tissue oxygen. Arch Surg 126(9): 1131-1134.

10. Peersman G, Laskin R, Davis J, Peterson M (2001) Infection in total knee replacement: a retrospective review of 6489 total knee replacements. Clin Orthop Relat Res 392: 15-23.

11. Crowe B, Payne A, Evangelista PJ, Stachel A, Phillips MS, et al. (2015) Risk Factors for Infection Following Total Knee Arthroplasty: A Series of 3836 Cases from One Institution. J Arthroplasty 30(12): 2275-2278.

12. Bozic KJ, Lau E, Kurtz S, Ong K, Berry DJ (2012) Patient-related risk factors for postoperative mortality and periprosthetic joint infection in medicare patients undergoing TKA. Clin Orthop Relat Res 470: 130137

13. McCleery MA, Leach WJ, Norwood T (2010) Rates of infection and revision in patients with renal disease undergoing total knee replacement in Scotland. J Bone Joint Surg Br 92(11): 1535-1539.

14. Harris AHS, Reeder R, Ellerbe L, Bradley KA, Rubinsky AD, et al. (2011) Preoperative alcohol screening scores: association with complications in men undergoing total joint arthroplasty. J Bone Joint Surg Am 93(4): 321-327.

15. Bradley KA, Rubinsky AD, Sun H, Bryson CL, Bishop MJ, et al. (2011) Alcohol screening and risk of postoperative complications in male VA patients undergoing major non-cardiac surgery. J Gen Intern Med 26(2): 162-169.

16. Schrama JC, Espehaug B, Hallan G, Engesaeter LB, Furnes O, et al. (2010) Risk of revision for infection in primary total hip and knee arthroplasty in patients with rheumatoid arthritis compared with osteoarthritis: a prospective, population-based study on 108,786 hip and knee joint arthroplasties from the Norwegian Arthroplasty Register. Arthritis Care Res (Hoboken) 62(4): 473-479.

17. Doran MF, Crowson CS, Pond GR, O Fallon WM, Gabriel SE (2002) Frequency of infection in patients with rheumatoid arthritis compared with controls: a population-based study. Arthritis Rheum 46(9): 22872293.

18. Bongartz T, Halligan CS, Osmon DR, Reinalda MS, Bamlet WR, et al. (2008) Incidence and risk factors of prosthetic joint infection after total hip or knee replacement in patients with rheumatoid arthritis. Arthritis Rheum 59: 1713-1720.
19. Momohara S, Kawakami K, Iwamoto T, Yano K, Sakuma Y, et al. (2011) Prosthetic joint infection after total hip or knee arthroplasty in rheumatoid arthritis patients treated with nonbiologic and biologic disease-modifying antirheumatic drugs. Mod Rheumatol 21(5): 469475 .

20. Krüger K, Albrecht K, Rehart S, Scholz R (2014) Recommendations of the German Society for Rheumatology on the perioperative procedure under therapy with DMARD and biologicals for inflammatory rheumatic diseases. Z Rheumatol 73(1): 77-84.

21. Wang Q, Goswami K, Shohat N, Aalirezaie A, Manrique J, et al. (2019) Longer Operative Time Results in a Higher Rate of Subsequent Periprosthetic Joint Infection in Patients Undergoing Primary Joint Arthroplasty. J Arthroplasty 34(5): 947-953.

22. Rohde JM, Dimcheff DE, Blumberg N, Saint S, Langa KM, et al. (2014) Health care-associated infection after red blood cell transfusion: systematic review and meta-analysis. JAMA 311(13): 1317-1326.

23. Borghi B, Casati A (2000) Incidence and risk factors for allogenic blood transfusion during major joint replacement using an integrated autotransfusion regimen. The Rizzoli Study Group on Orthopaedic Anaesthesia. Eur J Anaesthesiol 17(7): 411-417.

24. Müller MM, Meybohm P, Geisen C, Schmitz RT, Serve H, et al. (2014) Patient Blood Management - How does it work in practice? - The interdisciplinary cooperation. Anesthesiol Intensivmed Emergency Med Schmerzther 49(4): 266-272.

25. Koulouvaris P, Sculco P, Finerty E, Sculco T, Sharrock NE (2009) Relationship between perioperative urinary tract infection and deep infection after joint arthroplasty. Clin Orthop Relat Res 467: 18591867.

26. Saleh K, Olson M, Resig S, Bershadsky B, Kuskowski M, et al. (2002) Predictors of wound infection in hip and knee joint replacement: results from a 20-year surveillance program. J Orthop Res 20(3): 506515.

27. Poultsides LA, Ma Y, Della Valle AG, Chiu YL, Sculco TP, et al. (2013) In-hospital surgical site infections after primary hip and knee arthroplasty--incidence and risk factors. J Arthroplasty 28: 385-389.

\section{Your next submission with Juniper Publishers will reach you the below assets}

- Quality Editorial service

- Swift Peer Review

- Reprints availability

- E-prints Service

- Manuscript Podcast for convenient understanding

- Global attainment for your research

- Manuscript accessibility in different formats

( Pdf, E-pub, Full Text, Audio)

- Unceasing customer service

Track the below URL for one-step submission https://juniperpublishers.com/online-submission.php 